\title{
Explication du sens en classe de langue : le rôle du contexte
}

\author{
Delorme, Vera \\ Université Stendhal - Grenoble 3 \\ vera_delorme@yahoo.fr
}

\section{Explication du sens par le contexte}

Parmi les procédés que l'enseignant utilise en classe de langue pour expliquer le sens d'un élément langagier, Cicurel (1985) distingue la dénomination, la définition, la paraphrase et le recours au contexte. Notre étude porte sur ce dernier procédé. On commencera donc par préciser en quoi consiste l'usage des contextes dans la transmission du sens et quels sont les contextes utilisés.

Les pratiques par lesquelles s'effectue l'explication du sens par le contexte dans les interactions verbales en classe de langue se traduisent par la création dans et par le discours didactique des situations-exemples illustrant le sens d'un élément - langagier ou non langagier - à transmettre. Faisant partie de l'ensemble des pratiques de transmission employées par l'enseignant ${ }^{1}$, les pratiques en question, qui sont à la fois discursives et didactiques, consistent donc à construire un contexte fictionnel ou réel autour d'un mot d'une expression, d'un acte langagier ... - afin de créer " un nouveau « décor » au mot », de produire une «mise en intrigue, dans le but de faire comprendre l'usage du mot, de préciser qui dit ce mot, dans quelles circonstances » (Cicurel, 2002 : 180). Cette stratégie professorale permet de compenser le « déficit contextuel » (ibid.) en classe de langue et de fournir aux apprenants une « situation d'application légitime $»^{2}$ (Recanati, 2001) d'un élément lexical ou des « conditions d'appropriation contextuelle »d'un acte langagier (Kerbrat-Orecchioni, 2004). Le sens est ainsi entendu aussi bien en tant que signification linguistique qu'en tant que valeur pragmatique d'un énoncé.

Concernant la notion de contexte, on précisera qu'il s'agit ici de ce qu'on appelle généralement le contexte situationnel («externe » ou « objectif») qui peut être considéré comme « l'environnement réel, proche ou lointain des locuteurs, en fait tout ce qui n'est pas le message et notamment le message verbal » (Schmoll, $1996: 241)^{3}$. Il est à préciser également que le contexte situationnel possède un double statut en didactique des langues :

\begin{abstract}
«Dans l'enseignement/apprentissage des langues, les notions de contexte et de situation renvoient en premier lieu d'une part au cadre socio-institutionnel (enseignement secondaire, institution bilingue, etc.), d'autre part à l'univers de contenu des leçons et des méthodes (Rendez-vous à la gare, Faire connaissance, Achats dans les magasins, etc.). » (Porquier \& Py, 2004 : 50)
\end{abstract}

Dans l'analyse du discours didactique, les contextes situationnels (ou externes) sont donc d'une part les cadres où se déroulent les échanges (classe, institution), et d'autre part les situations qui sont introduites en classe de langue et qui «projettent, de façon plus ou moins directe, les contextes ultérieurs visés d'utilisation de la langue et d'interaction dans la langue, et souvent dans la culture, étrangères » (ibid. : 51). Cette dernière conception de la notion de contexte englobe également les situations-exemples crées ou évoquées dans le but d'expliquer le sens d'un élément langagier.

A travers l'analyse des transcriptions des interactions en classe de langue ${ }^{4}$, on observe une grande diversité des situations-exemples construites dans le discours. On peut ainsi distinguer :

- des contextes réels qui sont des situations existant ou ayant existé dans le monde, décrites et/ou évoquées par l'enseignant ;

- des contextes issus des objets textuels (par exemple, d'une œuvre littéraire) ; 
- des contextes fictionnels, parmi lesquels :

a) des « contextes fictionnels prototypiques », terme proposé par Cicurel (2002) pour désigner « un contexte virtuel [qui] a cependant pour caractéristique d'être "réaliste" ou vraisemblable. Il se veut représentatif de la classe d'événements narrés » (ibid.: 181);

b) des «fictions proximales » qui représentent des situations fictionnelles créées à partir du cadre actuel de l'interaction, donc celui de la classe ;

c) des «fictions-projections » - situations hypothétiques pouvant potentiellement devenir réelles dans le futur.

On notera également la diversité des moyens discursifs que les participants à l'interaction didactique utilisent dans la construction des contextes. On observe ainsi, dans tous les types de contextes, des procédés descriptifs ou encore certains traits narratifs (par exemple, discours rapporté, dialogué ou non, imaginaire ou non) mais aussi des moyens discursifs propres à tel ou tel type de situation et ayant des fonctions particulières, dont par exemple :

- les noms propres, permettant de reconnaître soit le domaine de référence où se situe le contexte évoqué, soit l'objet textuel dont il est issu ;

- l'auto- et/ou l'hétéro-fictionnalisation : procédé par lequel on place un ou plusieurs participants en présence - enseignant et/ou apprenant(s) - dans un contexte fictionnel ;

- les pronoms et/ou les noms « génériques » pour marquer la nature prototypique d'une situation.

\section{Situation-exemple}

Afin de mettre en lumière le fonctionnement des situations-exemples dans l'explication du sens en classe de langue on s'appuiera d'une part sur la notion de cadre de l'expérience développée par Goffman $(1974)^{5}$, et d'autre part sur la conceptualisation du contexte formulée en linguistique interactionnelle.

\subsection{Cadres naturels et sociaux}

Rappelons tout d'abord que Goffman (1974) distingue deux types de cadre : cadres naturels et cadres sociaux. Le cadre naturel est un événement physique qui est entièrement déterminé en tant que tel - «par la nature »- et n'exige aucun travail évaluatif. Autrement dit, pour interpréter ce type de cadre il suffit de faire appel à ses connaissances du monde. Et pour comprendre le sens de l'élément langagier placé dans ce type de contexte, il suffit de comprendre la description du cadre :

Extrait $1-$ «rossir » :

P : heu:: alors je cherche un exemple avec grossir ++ heu:: grossit +++++ la tempête + oui la tempête qui devient de plus en plus grande + au début il y a beaucoup de vent bon un petit peu et puis après ça devient monstrueux là on dira plutôt que la tempête a grossi + plutôt que s'est étendue

Cet extrait représente une réponse de l'enseignante à la question d'une apprenante formulée précédemment comme suit : " j'ai une question on a dit que:: vous vous avez dit que: le le + après l'union [se] grossit $\uparrow$ », " et pourquoi grossit à lieu de:: agrandir + ou est-ce qu'on peut choisir », "et généralement on pourrait utiliser le mot grossir pour quelque chose qui s'étend ». Afin de répondre à cette sollicitation de l'apprenante, l'enseignante cherche à créer une situation-exemple illustrant l'usage de l'élément langagier en question. L'extrait cité ci-dessus représente donc cette situation, celle de la tempête, qui est le cadre naturel et dont l'interprétation permettrait aux apprenants d'accéder directement au sens de l'élément lexical. 
A la différence du cadre naturel, l'interprétation d'un cadre social s'appuie, selon Goffman (1974), sur les « prémisses organisationnelles » associées à ce cadre. Autrement dit, lorsqu'un individu se trouve dans une situation donnée, il l'identifie en faisant appel à un ou plusieurs cadres ou schèmes interprétatifs qui sont « stockés » dans sa mémoire. Une fois la situation identifiée, l'individu va agir en conséquence selon les «principes d'organisation» du cadre. C'est ainsi que pour interpréter la situation évoquée dans l'extrait suivant, les apprenants sont invités à activer un schème interprétatif qu'ils ont dans leur mémoire :

Extrait 2 - « suspendre une loi » :

P : oui j'ai entendu j'ai VU ce je crois que c'est ce matin dans un journal que: il avait décidé de: de suspendre + vous savez il y a un processus comme dans aux Etats-Unis c'est la même chose hein $\uparrow+$ le gouvernement propose des lois + ok?+ ensuite les lois son:t données à discuter au Parlement

A : $\mathrm{mmh}$

$\mathrm{P}$ : à l'Assemblée Nationale d'abord + l'Assemblée Nationale discu:te ah oui c'est bien non on va changer ça on va changer ça + quand ils ont fini + ça passe au Sénat + le Sénat dit ce qui pen- hein oui ça c'est bien $\mathrm{mmm}+$ si le Sénat est d'accord avec l'Assemblée Nationale la loi revient au gouvernement et là on la vote ++ mais + si + le gouvernement propose une loi + et que la loi heu comme celle-ci heu soulève beaucoup beaucoup de: de: de: de colè:re et de: et de d'opposition + et bien le gouvernement peut dire bon je mets pas ma loi à discuter à l'Assemblée Nationale voi- et on appelle ça SUspendre sa loi + on suspend la loi + on attend

Le contexte (re)construit ici par l'enseignante est celui de l’ « Assemblée Nationale, Sénat». Ce contexte est introduit dans le discours dans le but d'illustrer le sens de «suspendre une loi ». Il est intéressant de remarquer par ailleurs que l'enseignante souligne ici explicitement le fait que le schème que possèdent déjà les apprenants est tout à fait applicable à la situation qu'elle évoque : «comme (...) aux Etats-Unis c'est la même chose ». Les « prémisses organisationnelles » permettent donc aux apprenants d'identifier le cadre social évoqué et de comprendre ainsi ce à quoi réfère «suspendre une loi ». L'interprétation de la situation livre alors la signification du vocable.

\subsection{Situation d'interaction}

Les extraits présentés ci-dessus (Extraits 1 et 2 ) ont un point commun : le contexte introduit dans le discours contient le référent de l'élément lexical à expliquer. L'extrait suivant illustre un cas de figure différent : ce n'est pas le référent mais l'occurrence de l'élément lexical à expliquer qui est placée dans le cadre construit :

Extrait 3 - « en toute bonne foi » :

$\mathrm{P}$ : par exemple si je vous dis heu:: la semaine prochaine l'université est fermée + bon + donc vous n'allez pas venir à l'université + et puis vous allez apprendre que: c'est pas vrai $\uparrow$ le: l'université était ouverte + il y avait des cours + donc vous allez venir me voir et dire mais: euh: qu'est-ce qui se pa:sse vous aviez dit heu: que c'était fermé: et puis en fait c'était pas fermé + et moi je vais dire mais en toute bonne foi $\uparrow$ je vous ai dit en toute bonne foi que c'était ou- c'était fermé + parce que je croyais vraiment que c'était fermé ++ hein $\uparrow$ donc c'est vraiment heu: j'ai pas: j'ai pas menti: j'ai pas: j'ai pas manipulé je je je le croyais moi aussi que c'était fermé

Afin d'illustrer l'expression « en toute bonne foi », l'enseignante crée un contexte fictionnel en utilisant sa propre identité et celles des apprenants ainsi que les éléments contextuels du cadre actuel de l'échange ( «l'université », « la semaine prochaine »). Les pronoms personnels « je » et « vous » se réfèrent ici à l'enseignante et aux apprenants qui sont placés dans une situation hypothétique et sont donc fonctionnalisés ${ }^{6}$. L'enseignante interprète ainsi son propre rôle - ainsi que celui des apprenants - et c'est dans une réplique de ce « je » fictionnel que l'expression à illustrer apparaît : « et moi je vais dire mais en toute bonne foi $\uparrow$ je vous ai dit en toute bonne foi que c'était ou- c'était fermé ». 
Ici, il ne suffit pas d'imaginer un cadre naturel (comme celui de la tempête) ou d'identifier un cadre social (comme celui de l'Assemblée Nationale). L'accès au sens repose, dans ce cas de figure, sur la détermination contextuelle du discours :

\begin{abstract}
«Le contexte est déterminant pour l'ensemble des choix discursifs : sélection des thèmes, formes d'adresse, niveau de langue, actes de langage (tributaires de leurs conditions de réussite, lesquelles sont de nature contextuelle), application des maximes conversationnelles (laquelle est également fonction du contexte, et en particulier des savoirs et intérêt que le locuteur attribue aux destinataires) : la prise en compte du contexte intervient à tous les niveaux. » (Kerbrat-Orecchioni, 2009 :17).
\end{abstract}

Les participants produisent donc leur discours en fonction des représentations qu'ils se font de la situation où ils se trouvent ; le discours est ainsi défini par le contexte dans lequel il se produit.

En classe de langue, lorsque l'enseignant introduit dans le discours une situation-exemple afin d'illustrer la signification linguistique, il s'appuie sur ce même principe de détermination contextuelle du discours mais en suivant le chemin inverse : le choix du contexte est déterminé par l'élément langagier à expliquer. Si pour les participants d'une situation de communication c'est le contexte qui les guide dans leur choix des éléments langagiers, ici c'est l'élément langagier qui va guider l'enseignant dans son choix de la situation. Autrement dit, le choix discursif est en quelque sort déjà «fait», il est «imposé » par la nécessité d'expliquer l'élément lexical, le travail de l'enseignante consiste alors à trouver dans le «stock» des contextes qu'elle a «dans sa tête », celui qui pourrait influencer ce choix. Le contexte est ainsi créé « sur mesure » pour rendre l'usage de l'élément langagier à expliquer le plus pertinent possible.

Pour les apprenants, afin d'accéder au sens de l'élément mis en contexte, il s'agit donc d'abord d'interpréter le cadre selon les "prémisses organisationnelles» qui leur permettent d'identifier la situation et ensuite d'établir un lien entre le contexte et le discours produit dans ce contexte. La signification de l'élément langagier est ainsi identifiée à travers les paramètres contextuels qui déterminent son choix : si l'on veut comprendre le sens de l'élément langagier mis en contexte, il faut comprendre comment le discours est influencé par les propriétés contextuelles évoquées ou décrites par l'enseignant ${ }^{7}$.

Le raisonnement se base alors sur les représentations que se font les locuteurs de la manière dont tel ou tel contexte définit le choix discursif, autrement dit, sur leurs connaissances préalables à propos de ce qui se dit habituellement dans une situation S. Par conséquent, une situation-exemple de ce type - donc contenant une occurrence de l'élément à transmettre - ne peut fonctionner en tant qu'outil de transmission que lorsqu'elle est suffisamment reconnaissable et représentative du lien existant entre le contexte et le discours. Cela implique - et explique - l'usage privilégié des contextes fictionnels prototypiques, dont on propose les exemples suivants :

Extrait 4 - « tout de même »:

A : et qu'est-ce que tout de même veut dire ?

$(\ldots)$

$\mathrm{P}:$ tout de même ?

An : oui

$\mathrm{P}:$ quand même malgré cela + tout de même + ah te voilà tout de même + tu attends quelqu'un: depuis longtem:ps + il est en reta:rd + et quand la personne arrive tu peux tu- ah te voilà quand même + te voilà tout de même + malgré le: bon malgré le fait que tu es: tu es en retard que j'ai attendu longtemps + tu tu arrives 
Extrait 5 - « un point c'est tout »:

$\mathrm{P}$ : vous entendez souvent les mamans ou les papas dire ça à leurs enfants tu vas te laver les mains $\uparrow$ avant de venir à table un point c'est tout ça veut dire discute pas ++ c'est pas la peine de discuter de dire ah: j'irai tout à l'heu:re ou je veux pas: un point c'est tout c'est pour fermer la discussion

Les deux situations représentées dans ces extraits sont donc à considérer comme les situations typiques de l'usage de la locution « tout de même » (Extrait 4) et de l'expression « un point c'est tout » (Extrait 5). Dans les deux extraits, les éléments langagiers à expliquer sont insérées dans le discours d'un personnage imaginaire mis en situation d'interaction ${ }^{8}$. L'identification de la situation se fait à la base des «prémisses organisationnelles » supposées « universelles » et donc faisant partie des schèmes interprétatifs que les apprenants possèdent déjà. La perception de l'influence des paramètres contextuels identifiés notamment du cadre participatif - sur le discours des personnages permet alors d'attribuer le sens aux éléments lexicaux mis en situation. On soulignera encore une fois que cette influence est ici particulièrement forte : la situation incite à produire l'élément langagier en question.

Il est à noter également que, dans certains cas, l'enseignant peut inviter les apprenants explicitement à établir le lien entre le contexte et le discours. Dans ce cas de figure, le discours professoral se présente sous forme de formule logique «si $S$, alors $X »$, où « $S$ » et « $X »$ sont respectivement la situation et le choix discursif approprié à cette situation :

Extrait 6 - « être débordé » :

$\mathrm{P}:(\ldots)$ je suis DEbordée vous comprenez? + je suis débordée? + par exemple si vous avez beaucoup de cou:rs et pour chaque cours beaucoup de travail à faire et des papiers à rendre vous pouvez dire j'en peux plus je suis DEbordée $+\mathrm{j}$ - TROP de travail à faire

La « formule » proposée ici par l'enseignante est donc la suivante : « si vous avez beaucoup de cou:rs et pour chaque cours beaucoup de travail à faire et des papiers à rendre » alors « vous pouvez dire j'en peux plus je suis DEbordée ». La situation décrite implique le choix de l'élément langagier à expliquer.

\section{Objets enseignés}

Notons tout d'abord que l'objet à transmettre en classe de langue représente non seulement le sens et/ou l'usage d'un élément langagier (un mot, une expression, une forme grammaticale...) mais aussi le contenu socioculturel - l'ensemble des connaissances extralinguistiques considérées comme associées à l'enseignement/apprentissage de la langue cible. A travers l'analyse de notre corpus, on observe que la mise en contexte peut être effectuée aussi bien pour la transmission d'un fait culturel que pour l'illustration de l'usage d'une forme grammaticale. Néanmoins, on constate que, dans notre corpus, le recours au contexte se fait le plus souvent lorsque l'objet d'enseignement est un élément lexical ou un acte de langage.

\subsection{Eléments lexicaux}

La construction des contextes dans le but de transmettre la signification des éléments lexicaux est illustrée par l'ensemble des exemples présentés ci-dessus. Les différents cas de figure observés peuvent être distingués en fonction de :

- type de cadre : naturel ou social ;

- type du contexte : fictionnel ou réel ;

- ce qui est mis en contexte : le référent de l'élément lexical à transmettre ou son occurrence.

Comme nous l'avons montré ci-dessus, dans tous ces cas de figure - à l'exception du cadre naturel l'accès au sens se fait par le recours à un schème interprétatif permettant d'identifier la situation et, si 
nécessaire, d'établir un lien entre le contexte et le discours. La transmission de la signification lexicale par le contexte exige donc que les apprenants aient dans leur répertoire les schèmes interprétatifs indispensables à l'identification de la situation proposée par l'enseignant. Le choix des contextes doit donc répondre à cette exigence (dans le cas contraire le procédé risque d'échouer). Par conséquent, on observe l'usage plutôt fréquent des situations prototypiques - donc facilement identifiables selon les «prémisses universelles » - ou encore des situations construites à la base du cadre de l'ici-maintenant (« fictions proximales »), donc celui de la classe.

On soulignera toutefois que, comme le constate Cicurel (1985), « les enseignants ont généralement recours à plusieurs procédés explicatifs à propos d'un signe inconnu » (id. : 35). La mise en contexte est donc souvent utilisée par l'enseignant avec d'autres procédés mis en œuvre pour expliquer le sens d'un élément langagier donné. L'analyse des extraits cités précédemment montre que, mise à part le recours à une situation, l'enseignant utilise également les procédés paraphrastiques (Extrait $6:$ «je suis DEbordée + j- TROP de travail à faire », Extrait $4:$ : quand même malgré cela + tout de même »; Extrait $2:$ « on suspend la loi + on attend », Extrait $5:$ : un point c'est tout ça veut dire discute pas »), la dénomination (Extrait 2 : « on appelle ça SUspendre sa loi ») et la définition (Extrait $5:$ « un point c'est tout c'est pour fermer la discussion $»)$.

L'usage par l'enseignant de plusieurs procédés simultanément s'explique selon Cicurel (1985) par le fait que :

\begin{abstract}
«(...) d'une part, il se peut, qu'en improvisant son explication, il [l'enseignant] ne parvienne pas tout de suite à mobiliser le type d'explication qu'il juge le plus adapté, d'autre part, les explications ne conviennent pas également à tous les membres du groupe-classe. Pour certains, la définition sera plus appropriée, pour d'autres ce sera la paraphrase ou le changement de contexte. » (ibid. : 35-36)
\end{abstract}

On ajoutera également que le recours à d'autres procédés, en même temps que la mise en contexte, permettrait de réduire le risque d'une éventuelle compréhension erronée que présente potentiellement l'usage des contextes dans l'explication du sens d'un élément lexical. Ce risque provient du fait que le contexte construit autour d'un mot ou d'une expression à expliquer permet de déduire le sens de l'élément langagier dans ce contexte. Il s'agit donc de l'interprétation pragmatique de l'élément lexical, le contexte étant une sorte de «filtre » qui ne laisse apparaître que certains aspects de la signification ${ }^{9}$ tout en dissimulant les autres :

\begin{abstract}
«Tout se passe comme si le sémème constituait une sorte de clavier (chaque touche représentant un sème) sur lequel le contexte joue. De la même manière que le pianiste n'appuie pas sur toutes les touches en même temps, le contexte actualise certains sèmes et neutralise les autres, en fonction du message qu'il est chargé de transmettre » (Galisson, $1970: 115$ )
\end{abstract}

On peut ainsi supposer qu'il existe un certain risque d'une éventuelle confusion chez l'apprenant dans le cas où ce dernier procède à une généralisation excessive. Le sens attribué à un élément $\mathrm{X}$ dans un contexte $\mathrm{S}$ peut être considéré par l'apprenant comme une propriété stable de $\mathrm{X}$ alors qu'il ne s'agit que de l'interprétation pragmatique, appropriée à $\mathrm{S}$, mais non appropriée dans un autre contexte. Ainsi par exemple en reprenant l'Extrait 4, il n'est pas exclu que l'apprenant associe la signification de « tout de même » à une situation de « reproche », ce qui peut provoquer un problème de compréhension lorsqu'il rencontrera ce même élément lexical dans un contexte différent de celui utilisé par l'enseignant; un malentendu est alors susceptible de survenir ${ }^{10}$.

Notons toutefois que, dans certains cas, afin d'illustrer plusieurs interprétations possibles d'un mot, l'enseignant peut le placer dans deux contextes différents. Ce procédé permet ainsi de montrer aux apprenants que le même élément langagier peut être interprété différemment en fonction de la situation où l'on utilise. L'extrait suivant illustre ce cas de figure : 
Extrait 7 - «beurs» (Activité en cours : lecture collective du texte de la chanson de MC Solaar « L'argent ne fait pas le bonheur »):

P: $(\ldots)$ on a dit que Marseille était une ville qui accueillait ++ beaucoup beaucoup d'immigrés + et en particulier des immigrés d'Afrique du Nord ++ et comme à Paris hein $\uparrow++$ généralement + ils se regroupent dans des quartiers qui sont plus défavorisés que les autres ++ les quartiers ici à Marseille c'est le Quartier Nord ++ et donc les gens + des jeunes de ce Quartier Nord sont donc d'origine + d'Afrique du Nord et donc ils sont de religion $\uparrow$

A1: musulmane

A2: arabe

P: musulmane + ils sont de religion musulmane + ce sont des + ce qu'on: heu: souvent en France aujourd'hui on: on les appelle alors + c'est une expre- c'est une expression qui a Passé VRAIment dans dans le langage courant + qui n'est pas forcément toujours acceptée hein $\uparrow$ par par les jeunes + on les appelle les beurs (va au tableau) les beurs (écrit au tableau) c'est une: transformation + heu: du mot arabe en verlan hein $\uparrow$ rebeu qui est devenu ensuite beur ++ d'accord? + donc c'est pour ça que c'est pas toujours une appellation qui est: heu: qui est: agréable pour pour eux + mais qui est vraiment passée dan:s dans le langage français vous le trouverez vous la trouverez même dan:s dans les journaux souvent (...)

Le contexte (réel) évoqué ici est celui de la ville de Marseille, et plus précisément du Quartier Nord de Marseille, dont certaines caractéristiques déjà introduites dans le discours sont rappelées par l'enseignante au début de l'extrait : on a dit que Marseille était une ville qui accueillait (...) beaucoup d'immigrés + et en particulier des immigrés d'Afrique du Nord ++ et comme à Paris hein $\uparrow++$ généralement $+i l s$ se regroupent dans des quartiers qui sont plus défavorisés que les autres ++ les quartiers ici à Marseille c'est le Quartier Nord ++ et donc les gens + des jeunes de ce Quartier Nord sont donc d'origine + d'Afrique du Nord. La sollicitation professorale que l'on observe ensuite - et donc ils sont de religion $\uparrow-$ permet aux apprenants de participer à la construction du contexte en formulant les réponses suivantes : musulmane (A1); arabe (A2), dont une est validée par l'enseignante : musulmane + ils sont de religion musulmane. Suit alors une définition commençant par le présentateur " ce sont » qui amène l'introduction d'un élément langagier nouveau pour les apprenants. Le mot «beurs » sera alors présenté aux apprenants par l'enseignante qui essayera d'expliciter les valeurs sociales et affectives qui sont inscrites dans la signification de ce mot et donc les conditions appropriées de son usage en contexte.

La présentation de cet élément langagier nouveau se développe ainsi dans le discours professoral qui suit. On remarque tout d'abord un grand nombre de pauses, d'hésitations et de « faux départs » qui traduisent probablement l'incertitude de l'enseignante quant à l'explication qu'elle propose. On observe ensuite l'explicitation des valeurs de ce mot qui, selon l'enseignante, varient d'un contexte à l'autre. Ainsi, en dehors du contexte du "Quartier Nord», le mot «beur» serait fréquent, largement diffusé et «standard»: «souvent en France aujourd'hui on: on les appelle », «c'est une expression qui a PAssé VRAIment dans dans le langage courant », « qui est vraiment passée dan:s dans le langage français vous le trouverez vous la trouverez même dan:s dans les journaux souvent ». En revanche, dans un contexte où les locuteurs sont ceux qui sont désignés par «beurs », l'élément langagier en question peut avoir un aspect «menaçant» leur face : «c'est pas toujours une appellation qui est: heu: qui est: agréable pour pour eux », « qui n'est pas forcément toujours acceptée hein $\uparrow$ par par les jeunes ${ }^{11}$. L'explication du sens de l'élément lexical en question prend ainsi forme d'une « mise en garde » implicite concernant son usage dans certains contextes.

\subsection{Actes langagiers}

Si dans une situation prototypique les comportements langagiers déclenchés par les paramètres contextuels sont prévisibles, le cas est différent lorsqu'il s'agit d'un cadre dont les principes d'organisation sont particulièrement variables en fonction de l'appartenance des locuteurs à tel ou tel groupe socioculturel ${ }^{12}$. En classe de langue, les «prémisses organisationnelles » propres à la langue et la 
culture enseignées sont ainsi à considérer comme les "schèmes interprétatifs cible » faisant partie de l'ensemble des règles pragmatiques à acquérir :
«De même que l'acquisition de la langue maternelle inclut celle des règles pragmatiques, de même ces règles doivent-elles être enseignées à ceux qui apprennent une langue étrangère, car elle font partie de plein droit de la « compétence » des sujets parlants. Plus précisément :
(1) Enseigner une langue, c'est enseigner aussi le fonctionnement des actes de langage, c'està- dire un ensemble de règles de corrélations entre des structures formelles et des valeurs illocutoires.
(2) Enseigner les actes de langage c'est enseigner aussi leurs utilisations, c'est-à-dire un ensemble de règles de corrélations entre des emplois et des conditions d'emploi. S'il est nécessaire de connaitre les formules de remerciement, encore faut-il savoir dans quelles circonstances il convient de remercier, et dans quels cas il vaut mieux s'en abstenir. S'agissant de même des structures interrogatives : s'il importe de savoir comment elles sont fabriquées, encore faut-il connaître les circonstances qui vont leur donner une valeur de question, de requête ou de salutation, et quelles sortes de questions sont attendues, permises ou exclues dans telle ou telle situation communicative - faute de quoi on s'expose à toutes sortes de déboires dès qu'on se trouvera en contact avec un étranger (...)» (Kerbrat-Orecchioni, 2002: 8, l'auteure souligne)

A travers l'analyse des interactions en classe de langue, on observe que le recours au contexte peut être pratiqué pour enseigner aussi bien les règles de corrélations entre des structures et des valeurs (voir l'Extrait 5 ci-dessus) que celles de corrélations entre des emplois et des conditions d'emploi. On s'intéressera en particulier à ce dernier cas de figure où l'on distinguera les pratiques de contextualisation qui visent l'enseignement des valeurs que peut prendre telle ou telle formulation dans un contexte donné, et d'autre part la mise en contexte qui illustre les circonstances appropriées à la production de tel ou tel acte.

\subsubsection{Formulations et valeurs}

Extrait $8-$ « répondre à un compliment » :

$\mathrm{P}:$ (...) avez-vous remarqué comment les Français reçoivent les compliments? + si vous dites à quelqu'un mmm qu'est-ce que ton pull est: est joli ++ ou bien heu:: c'était vraiment bien le livre que tu m'as recommandé + j'ai beaucoup aimé le livre que tu m'as offert ou: + qu'est-ce qu'est-ce que répondent souvent les Français? + quand on leur fait un compliment sur eux-mêmes + ah tu es bien coiffée + tu as un joli manteau ++ et bien souvent ils répondent en en minimisant vous comprenez minimisant? minimiser? par exemple on vous dit ah tu t'es bien coiffée aujourd'hui on va dire oh non (imitation gestuelle et vocale) pff c'est juste un brushing: c'est rien + oh non je suis affreu:se (rire) pour dire ça + ou bien ah t'as un joli manteau: + non pff ça fait longtemps que je l'ai ce manteau-là tu l'avais jamais vu: + bon il y a une sorte de: de refus du: du du compliment + eh: par exem- 'fin moi c'est avec les étudiants américains et avec mes collègues américaines que j'ai appris à à: à dire merci quand on me faisait un compliment + maintenant je dis merci (rire)

Les contextes que l'enseignante construit ici illustrent «comment les Français reçoivent les compliments », autrement dit, la réalisation - culturellement marquée - de l'acte de répondre à un compliment en français et dans la société française. Les compliments fictifs sont d'abord adressés aux personnages imaginaires : «si vous dites à quelqu'un mmm qu'est-ce que ton pull est: est joli ++ ou bien heu:: c'était vraiment bien le livre que tu m'as recommandé + j'ai beaucoup aimé le livre que tu m'as offert (...) ah tu es bien coiffée + tu as un joli manteau ». Ensuite, l'enseignante crée des échanges entre les personnages fictifs afin d'illustrer le fait que « les Français (...) quand on leur fait un compliment sur eux-mêmes (...) bien souvent ils répondent en en minimisant ». Les compliments fictionnels formulés auparavant par l'enseignante sont ainsi repris par elle-même et introduits dans les échanges fictionnels : « on vous dit ah tu t'es bien coiffée aujourd'hui on va dire oh non (imitation gestuelle et vocale) pff c'est juste un brushing: c'est rien + oh non je suis affreu:se ». Ce sont donc ces formulations « minimisées »- 
construites par la négation - présentées comme caractéristiques de la culture française, qui sont illustrées et transmises à travers la situation fictionnelle mise en scène par l'enseignante.

L'ensemble des énoncés-réponses est désigné par l'enseignante comme : « une sorte de: de refus du: du du compliment ». Le « refus » est donc présenté en tant que réponse conventionnelle à un compliment : il il a une valeur d'une réponse tout à fait «polie » et ne comporte aucun aspect menaçant pour la face de l'interlocuteur. Il est à remarquer également que la réponse à un compliment par le « refus » est mise en opposition avec le remerciement, ce dernier étant considéré par l'enseignante comme propre à la culture des apprenants (qui sont tous de nationalité américaine). La comparaison des deux comportements langagiers possibles dans la même situation permet ainsi aux apprenants de percevoir la différence culturelle.

L'extrait suivant, issu de la même séquence, va dans le même sens : la situation illustre l'usage de la locution «pas mal » dans un contexte qui lui donne une valeur d'appréciation (fortement) positive :

Extrait 9 - « donner une appréciation positive » :

$\mathrm{P}:(\ldots)$ très souvent les Français minimisent + par les choses comme oh pff non c'est un vieux truc heu: ah ouf pas ma:l ou non je trouve pas enfin bon c'est jamais merci: je suis conten:te + etcetera $m m h \uparrow(\ldots)$

$\mathrm{P}:(\ldots)$ les Parisiens surtout + plus que les provinciaux theu: ont du mal à: à: à accepter les choses positives + même dans leurs commentaires+ par exemple vous êtes allé voir un film + et: vraiment un très bon film vous avez beaucoup aimé votre amie française aussi elle a beaucoup aimé +et puis après vous allez en parler à d'autres personnes + et on va lui demander à votre amie française alors le film c'était bien? ah ouais pas mal (petits rires des apprenants) + elle va répondre pas mal (va au tableau) + c'était pas mal + au lieu de dire oui vraiment c'était c'était bien + c'est un bon film + elle dit oh + c'est pas mal + et souvent avec heu: + heu: des des gestes d'appréciation hein $\uparrow$ ah ouais c'était PAS mal hein $\uparrow+$ hein $\uparrow+$ mais on préfère cette formulation négative + plutôt qu'une formulation positive

Le contexte construit illustre ici « le fait que: + les Parisiens surtout + plus que les provinciaux (...) ont du mal à: (...) accepter les choses positives + même dans leurs commentaires ». Le groupe socioculturel est donc défini. Par la suite, l'enseignante introduit - par « par exemple » - une situation fictionnelle suivante : « vous êtes allé voir un film + et: vraiment un très bon film vous avez beaucoup aimé votre amie française aussi elle a beaucoup aimé + et puis après vous allez en parler à d'autres personnes + et on va lui demander à votre amie française alors le film c'était bien? ». On obtient ainsi un personnage dont l'identité culturelle est clairement définie et qui est placé dans une situation donnée. C'est donc en fonction de cette situation que le personnage «produit» un énoncé avec la locution «pas mal»: « ah ouais pas mal $(\ldots)+$ elle va répondre pas mal $(\ldots)+$ c'était pas mal ». Cette action langagière est ensuite comparée avec une autre, «alternative » : « au lieu de dire oui vraiment c'était (...) bien + c'est un bon film + elle dit oh + c'est pas mal + et souvent avec $(\ldots)$ des gestes d'appréciation hein $\uparrow$ ah ouais c'était PAS mal ». Ici encore, deux comportements sont ainsi mis en comparaison afin de rendre plus saillante la réalisation - considérée comme conventionnelle - de l'acte en question dans la langue et la culture cible.

\subsubsection{Conditions d'emploi}

Extrait 10 - «saluer » (Activité en cours : discussion autour d'un texte présentant certaines « règles de conduite dans la société française ». Au début de la séquence, l'enseignante demande aux apprenants quelles sont les « règles » qui leur semblent «bizarres » et pourquoi. En réponse à cette sollicitation professorale, l'apprenante A1 cite deux « règles » formulées ainsi : « ne vous promenez pas le sourire aux lèvres, tout le monde pensera que vous êtes un handicapé mental» et «ne regardez pas les personnes directement dans les yeux ». L'extrait présenté ci-dessous commence après la citation de la deuxième « règle ») :

A1: oui eh:: + pour moi + moi à l'université où j'allais $+\mathrm{c}$ - c'est normal $\uparrow$ on regarde les personnes et on a: + on dit bonjour + on sourit 
P: oui mais et dans la rue ici quand tu regardes quelqu'un tu lui dis bonjour + tu souris $\uparrow+$ et tu lui regardes aussi dans les yeux

A1: on peut?
P: oui oui oui

A1: ah ok (petit rire) je ne savais pas (petit rire)

P: par exemple quand tu entres dans l'université ici en bas + il y a le gardien par exemple

A1: ah oui

P: bon peut-être les étudiants ne le font pas mais les professeurs le font $\uparrow+$ heu on rentre on regarde le monsieur on on lui fait un sourire on lui dit bonjour $\uparrow+$ c'est ça accompagne: l'acte de: de politesse + mais:: c'est pas quelque chose qu'on fait dans la rue + si on croise quelqu'un qu'on::

A1: qu'on ne connaît pas

P: avec le- avec qui on n'a pas envie d'avoir un un contact on n'a pas besoin d'avoir un un contact

A1: oui c'est vrai

P: donc c'est vrai qu'on ne regardera pas dans les yeux et on ne sourira pas

A1: oui c'est peut-être $=$

$\mathrm{P}:=$ on va se croiser comme ça

Les échanges portent donc sur les conditions d'emploi des actes - langagier et non langagiers ${ }^{13}-$ qui $^{\prime}$ semblent être différentes dans la société française et dans la société américaine : selon les interactants, la production de l'acte de salutation "dans la rue » ou "à l'université » est tout à fait possible aux EtatsUnis et cela sans aucune condition, et elle est exclue en France «si on croise quelqu'un (...) avec qui on n'a pas envie d'avoir un un contact on n'a pas besoin d'avoir un un contact $\gg$. Les conditions d'emploi des actes en question dans la société française sont illustrées par l'enseignante à travers la situation de contact avec le "gardien à l'université » (avec toutefois une remarque sur la variation "interne » entre les «professeurs » et les « étudiants »).

Dans la suite de l'extrait, d'autres différences émergent :

A1: c'est peut-êt- je trouve que + c'est peut-être un peu bizarre parce que peut-être on + on dit bonjour à quelqu'un qu'on ne connaît pas peut-être dans la rue ou dans l'université ou quelque chose comme ça + mais: dans l'ascenseur + on ne se:: salue pas $\uparrow$ c'est ç- on dit XXX comme ça? ou:

P: on ne se salue pas? tu veux dire ici en France?

A1: oui non non non aux Etats-Unis

$\mathrm{P}: \mathrm{ah}$

A1: peut-être + je sais pas + (se tourne vers A3) quand tu entres + tu entres dans l'accenteur + tu dis: quelque chose?

\section{A3: XXX}

A1: elevator

A3: ah: non

A1: on ne: on ne dit pas bonjour mais ici c'est c'est plutôt vrai si tu es

P: oui ici généralement quand on entre dans l'ascenseur + on dit bonjour + quand on sort on dit au revoir bonne journée 
Les conditions d'emploi de la salutation mentionnées ici sont donc totalement opposées dans la culture « source » et la culture « cible » : aux Etats-Unis « on dit bonjour à quelqu'un qu'on ne connaît pas peutêtre dans la rue ou dans l'université ou quelque chose comme ça + mais: dans l'ascenseur + on ne se:: salue pas » et c'est exactement le contraire en France. On observe ainsi l'explicitation, par le contexte, des « prémisses organisationnelles » permettant d'identifier une situation comme appropriée ou non à la production de l'acte de salutation. Notons par ailleurs qu'il s'agit ici également de la prévention des malentendus potentiels pouvant survenir dans une situation de contact interculturel ; le malentendu étant représenté comme résultant d'une divergence entre les interprétations (Laforest \& Vincent, 1999) et expliqué par la variation interculturelle dans le fonctionnement des actes de langage.

On soulignera également que l'on observe la construction discursive des contextes est effectuée ici en commun par plusieurs participants à l'interaction didactique. Les conditions d'emploi de la salutation sont ainsi mises en discours collectivement à travers les échanges et comparées d'une culture à l'autre. On parlera donc de la co-construction du sens : le sens que les participants donnent à la situation même ainsi qu'à l'action verbale qui lui est associée. Le discours produit en commun donne ainsi naissance à une représentation partagée, qui se construit et se précise tout au long des échanges, sur les différences au niveau de l'emploi de l'acte en question dans la culture « source » et la culture « cible ».

L'ensemble des divergences culturelles, relevées lors des échanges entre l'enseignante et les apprenants, est explicité dans le discours professoral à la fin de la séquence :

$\mathrm{P}:$ donc $+\mathrm{c}^{\prime}$ est très intéressant ces ces ces ces différences parce que ça vous montre pourquoi + parfois il peut y avoir des malentendus + hein $\uparrow+$ entre des gens des cultures différentes + pourquoi une attitude qui est naturelle pour vous peut déclencher des réactions heu:: que vous ne comprendrez pas +++ et: le sourire et: surtout si vous souriez franchement heu:: heu: bon à: tout le monde + et: en particulier dans les relations qui sont très codifiées entre les hommes et les femmes + là effectivement + si vous souriez à un homme ici en France d'une manière + très franche très directe il va pas du tout l'interpréter comme ah elle est heureuse elle est contente il va dire tiens elle me drague

As: (rire)

P: elle me drague (rire) et après bon évidemment il va se croire autori- c'est ça qui t'est arrivé?

Cette dernière mise en contexte nous semble particulièrement intéressante car le processus interprétatif prototypique et propre à la langue et à la culture cible - est mis en discours par l'enseignante de manière explicite. Le contexte construit ici est le contexte fictionnel : "si vous souriez à un homme ici en France d'une manière + très franche très directe il va pas du tout l'interpréter comme ah elle est heureuse elle est contente il va dire tiens elle me drague (...) et après bon évidemment il va se croire autori- ». Le processus interprétatif - prototypique - d'un «homme ici en France» est mis en discours de l'enseignante de manière explicite. L'accent est mis ainsi sur la détermination culturelle de l'interprétation de la situation évoquée. Autrement dit, le discours professoral illustre ici la manière dont les « prémisses organisationnelles », culturellement marquées, « fonctionnent » dans la situation donnée. C'est donc ce schéma interprétatif qui constitue ici l'objet de transmission. Et c'est à travers cette illustration que les apprenants accèdent au sens de l'acte, plus précisément à la valeur que cet acte prend habituellement dans la situation décrite.

\section{Conclusion et perspectives}

L'explication du sens par le contexte se réalise à travers la mise en discours d'un certains nombre de paramètres contextuels (lieux, participants, finalités) qui permettent d'interpréter un élément langagier inconnu. Cette interprétation se base sur les schèmes interprétatifs, supposés partagés, que l'individu l'apprenant dans notre cas - possède dans sa mémoire. 
Les schèmes interprétatifs, ou, dans les termes de Goffman (1974), les " prémisses organisationnelles » des cadres sociaux jouent ainsi un rôle crucial dans la transmission du sens à travers la (re)construction discursive des contextes. D'une part, ils représentent les mécanismes qui permettent d'interpréter une situation construite et de ce fait, d'accéder au sens d'un élément à transmettre, qu'il soit inséré dans cette situation ou représenté par celle-ci. D'autre part, ces schèmes interprétatifs, en tant que constituants d'une culture, peuvent eux-mêmes faire partie du contenu à enseigner/apprendre « ciblé ». Enfin, dans la mesure où toute situation comprenant une action quelconque - langagière ou non - est déterminée par des principes organisationnels, et que l'ensemble des cadres sociaux d'un groupe donné est selon Goffman au centre de sa culture, on peut supposer que toute situation de ce genre, (re)construite dans le discours de la classe, véhicule inévitablement une part du culturel. Dans ce sens, les schèmes interprétatifs associés à telle ou telle situation peuvent être considérés comme des objets de transmission "invisibles » qui «se glissent » dans la construction d'un contexte lorsque ce dernier est mis au service de la transmission du sens.

En outre, et cela nous semble particulièrement intéressant, à travers l'analyse des extraits du corpus et en nous appuyant sur la conceptualisation du contexte en linguistique interactionnelle, nous constatons que le contexte construit dans et par le discours didactique dans le but de transmettre le sens d'un élément langagier reflète le fonctionnement d'une situation d'interaction, telle qu'elle existe en dehors de la classe. Attribuer un sens à un élément langagier inconnu produit dans une situation réelle (par exemple lors d'une consultation médicale ou un cours de judo) c'est faire appel à ses représentations sur le lien entre les paramètres contextuels et le discours. Attribuer un sens à un élément langagier nouveau, placé dans une situation évoquée, racontée, décrite dans le discours de la classe de langue, suppose la même démarche. Par conséquent, la transmission du sens par le contexte reflète le processus de la construction du sens dans une situation d'interaction. Si le discours est influencé par les propriétés contextuelles, la construction d'un contexte dans le discours de la classe s'appuie sur la représentation que se font les participants de cette influence. La détermination contextuelle du discours permet ainsi d'utiliser le contexte en tant qu'outil de l'explication du sens dans les interactions verbales en classe de langue.

On remarquera également que, comparée à d'autres procédés d'explicitation du sens, la mise en contexte semble être le seul procédé réalisable uniquement dans et par le discours de la classe. Autrement dit, à la différence des co-textualisations que l'on peut retrouver dans des dictionnaires ou encore de l'extraction des éléments langagiers d'un contexte «commun » que proposent généralement les méthodes de langue, c'est seulement dans le discours de la classe que le sens à transmettre peut être explicité à travers un contexte dont les propriétés sont assemblées expressément pour un objet donné et forment ainsi pour ce dernier un ensemble « sur mesure » qui, en outre, est conçu aussi en fonction du public. On soulignera donc cette complémentarité qui existe en matière de transmission du sens entre les supports d'enseignement/apprentissage et le discours de la classe.

Le dernier point que l'on voudrait évoquer concerne l'efficacité de l'explication du sens par le contexte du point de vue de l'apprentissage. Rappelons tout d'abord que ce procédé est rarement utilisé par l'enseignant sans avoir recours en même temps à d'autres procédés, tels que par exemple la paraphrase ou la définition. Il semble donc problématique d'établir - sans modifier le cadre des échanges - une corrélation entre un éventuel réemploi d'un élément langagier par les apprenants et sa mise en contexte dans le discours de la classe. Néanmoins, il nous parait pertinent d'insister sur deux aspects de l'explication du sens par le contexte qui pourraient éventuellement offrir des opportunités pour l'apprentissage. Le premier relève du fait que les contextes peuvent être construits en commun par les participants à l'interaction, ce qui permet ainsi de considérer les pratiques didactiques en question comme pouvant donner lieu à la co-construction du sens, et donc ayant un potentiel appropriatif. Le deuxième concerne l'explicitation des liens entre le contexte et l'interprétation/production du discours que l'on peut observer lors de l'explication du sens. Le recours à une situation offre ainsi non seulement l'accès au sens mais aussi l'accès à l'information métapragmatique. Il s'agit alors de l'enseignement explicite de règles pragmatiques, susceptible d'apporter une éventuelle contribution au développement de la compétence pragmatique chez l'apprenant. 


\section{Références bibliographiques}

Bateson, G. (1955). Une théorie du jeu et du fantasme, in Vers une écologie de l'esprit, 1977, Paris : Le Seuil, 209224.

Cicurel, F. (1985). Parole sur parole ou le métalangage dans la classe de langue. Paris : CLE International.

Cicurel, F. (2002). Les réagencements contextuels dans l'enseignement des langues, dans Cicurel F., Véronique D. (éds), Discours, action et appropriation des langues, Paris : PSN.

Cicurel, F. (2005). La flexibilité communicative : un atout pour la construction de l'agir enseignant. Le français dans le monde. Recherches et Applications, Paris : CLE International. 180-191.

Filliettaz, L. (2006). La place du contexte dans une approche praxéologique du discours. Le cas de l'argumentation dans les interactions scolaires, Pratiques, Numéro thématique "Textes et contextes", Université de Metz, 71-88.

Galisson, R. (1970). Analyse sémique, actualisation sémique et approche du sens en méthodologie, Langue française $8,107-116$

Goffman, E. (1974). Les cadres de l'expérience ; trad. par I. Joseph, M. Dartevelle \& P. Joseph, 1991, Paris Les Editions de Minuit.

Kerbrat-Orecchioni, C. (1990). Les interactions verbales, Tome I, Paris : A. Colin.

Kerbrat-Orecchioni, C. (2002). Présentation, in Kerbrat-Orecchioni, C. \& Traverso, V. (éds) : Actes du Symposium "Variations culturelles dans les comportements communicatifs", Congrès de l'ARIC, http://www.unige.ch/fapse/SEE/groups/aric/Actes.htm

Kerbrat-Orecchioni, C. (2004). «Que peut-on faire avec du dire?» Cahiers de Linguistique Française 26 «Les modèles du discours face au concept d'action », 27-43.

Kerbrat-Orecchioni, C. (2009). Le traitement du contexte en analyse du discours en interaction, dans Analyses du discours et contextes, textes réunis et présentés par Sandré, M. Éditions Lambert-Lucas, Limoges, 11-30

Laforest, M. \& Vincent, D. (1999). Incompréhension et malentendu: deux manifestations de la co-construction du sens, Langues et linguistique, $\mathrm{n}^{\circ} 25,111-144$.

Moirand, S. (2007). Discours, mémoires et contextes : à propos du fonctionnement de l'allusion dans la presse, Corela, Numéros spéciaux, Cognition, discours, contextes. Disponible en ligne : http://corela.edel.univpoitiers.fr/index.php?id=1567.

Porquier R. \& Py, B. (2004). Apprentissage d'une langue étrangère : contextes et discours, Paris : Didier, Coll. Credif Essais.

Recanati, F. (2001). Déstabiliser le sens, Revue Internationale de Philosophie, 2, 197-208. 179-194.

Schmoll, P. (1996). Production et interprétation du sens : la notion de contexte est-elle opératoire ?, Scolia, 6, 235255

Schütz, A. (1945). On Multiple Realities, Collected Papers, vol. I : The Problem of Social Reality, 1962, La Haye: Martinus Nijhoff, 205-259. Traduction française: Sur les réalités multiples, Le chercheur et le quotidien: phénoménologie des sciences sociales, trad. par A. Noschis-Gilléron, 1987, Paris, Klincksieck, 103-167.

Sperber, D. \& Wilson, D. (1986). La pertinence: communication et cognition, trad. par A. Gerschenfeld et D. Sperber, 1989, Paris, Les Éditions de Minuit.

Vion, R. (1992). La communication verbale : analyse des interactions, Paris Hachette supérieur. 


\begin{abstract}
${ }^{1}$ Le terme "pratiques de transmission» est proposé par Cicurel (2005) et désigne : «des pratiques langagières (verbales et non verbales) et des pratiques interactionnelles qu'un enseignant met en œuvre afin qu'un public moins savant puisse s'approprier des savoirs et des savoir-faire » (ibid. : 185).

${ }^{2}$ Selon Recanati (2001), le potentiel sémantique d'un prédicat $\mathrm{P}$ peut être conçu comme une collection de situations où l'application de $\mathrm{P}$ est jugée légitime par les membres de la communauté ; ainsi apprendre $\mathrm{P}$ consisterait pour l'apprenant à enregistrer un certain nombre de situations qui légitiment l'application de P.
\end{abstract}

${ }^{3}$ Voir également les définitions proposées par Vion (1992) et Kerbrat-Orecchioni (1990).

${ }^{4}$ Corpus des transcriptions des interactions en classe de français langue étrangère ; Cours organisés pour les étudiants américains à Paris, dans les locaux d'une université; professeur : enseignante expérimentée de FLE, locutrice native. Conventions de transcription: «+» pause, «: ) allongement de la syllabe, " $\uparrow$ » intonation montante, «XXX» inaudible, «=» enchaînement rapide, mots en italiques = mots en langue étrangère, mots en italiques entre parenthèses $=$ commentaire sur le non-verbal, mots en majuscules $=$ emphase intonative, mots soulignés $=$ chevauchement ; $\mathrm{A}=$ apprenant, $\mathrm{P}=$ professeur, $\mathrm{An}=$ apprenant non-identifié.

${ }^{5}$ Le concept goffmanien se base sur les notions de «cadre psychologique » de Bateson (1955) et de «province limitée de signification» de Schütz (1945). Les notions de cadre de l'expérience, de cadre psychologique et de «province limitée de signification» représenteraient toutes un «contour » virtuel qui délimite une classe ou un ensemble d'expériences, d'actions, d'évènements et qui oriente l'interprétation de ses contenus. Chez Goffman (1974) le terme de cadre est défini comme suit : « Je soutiens que toute définition de situation est construite selon des principes d'organisation qui structurent les événements - du moins ceux qui ont un caractère social - et notre propre engagement subjectif. Le terme de « cadre » désigne ces éléments de base. » (ibid. : 19)

${ }^{6}$ On a ici un exemple d'un «contexte fictionnel proximal» créé à travers la fonctionnalisation des éléments de la situation de l'ici-maintenant (locuteurs, lieu, temps); le cadre d'illustration virtuel est construit sur la base de l'existant.

${ }^{7}$ Parmi les paramètres contextuels, on distinguera suivant Kerbrat-Orecchioni (1990), le cadre spatio-temporel, les participants et les buts de l'interaction. A travers l'analyse de notre corpus, on observe que la construction d'un contexte dans le but d'expliquer le sens d'un élément langagier peut être effectuée par la mise en discours d'une partie ou de l'ensemble de ces « ingrédients » d'une situation d'interaction. On notera également que les paramètres contextuels peuvent être plus ou moins déterminés et plus ou mois spécifiés dans le discours.

${ }^{8} \mathrm{Ce}$ discours est à considérer comme un cas de discours rapporté dialogué.

${ }^{9}$ A savoir, ceux qui permettent d'aboutir à l'interprétation la plus pertinente ; la pertinence étant entendue dans sa conception développée dans le cadre de la théorie de la pertinence de Sperber \& Wilson (1986). Selon la théorie de la pertinence, le calcul inférentiel, que le récepteur effectue afin de construire une hypothèse sur le sens voulu d'un énoncé, est guidé par ses considérations de pertinence. La pertinence est définie en termes d'effets cognitifs et efforts mentaux : la sélection de l'hypothèse la plus pertinente pour l'individu se fait en fonction du rapport entre les efforts qu'il fait dans le traitement d'un input et les effets cognitifs positifs entraînés par ce traitement.

${ }^{10}$ Il nous semble intéressant d'évoquer ici également la question de la réflexivité du contexte dont on rappellera l'idée générale : «Le discours façonne le contexte autant que le contexte façonne le discours : c'est là une idée qui apparaît aujourd'hui dans la littérature comme un véritable leitmotiv. » (Kerbrat-Orecchioni, 2009: 16). Dans la mesure où le discours a cette "capacité » de reconfigurer les propriétés contextuelles, une confusion dans l'attribution du sens à un élément langagier peut avoir une conséquence sur l'interprétation de la situation dans laquelle cet élément est produit. Le malentendu pourra alors avoir plusieurs sources successives : interprétation erronée d'un élément langagier $\rightarrow$ interprétation erronée de la situation $\rightarrow$ interprétation erronée du discours produit dans cette situation....

${ }^{11}$ Il est intéressant de remarquer ici que, selon l'enseignante, la valeur affective pouvant être attribuée au mot «beurs » par certains locuteurs s'explique par l'étymologie : "c'est une: transformation + heu: du mot arabe en verlan hein $\uparrow$ rebeu qui est devenu ensuite beur ++ d'accord? + donc c'est pour ça ». En effet, le mot en question fait partie de ce que Moirand (2007) désigne comme «mots porteurs de mémoire ». Il s'agit de la mémoire collective qui est inscrite dans le mot et «qui n'est pas la même pour tous les groupes sociaux selon le degré d'implication dans l'événement ». Les valeurs que l'on peut attribuer à l'élément lexical en question sont donc extrêmement variables, non seulement en fonction du contexte (et du cotexte) mais aussi en fonction des souvenirs, des représentations, du 
sentiment d'appartenance à un groupe social, de l'histoire personnelle, des attitudes individuelles, etc. d'un locuteur donné.

${ }^{12}$ Dans la conception de Filliettaz (2006), toute situation d'action est à envisager « à l'intersection d'une double logique de façonnage, à savoir comme configurée à la fois de l'extérieur, par des préconstruits à caractère sociohistorique qui préexistent à la production du discours, et de l'intérieur, par les mécanismes discursifs eux-mêmes » (ibid. : 77).

${ }^{13}$ Il s'agit ici des actes non langagiers « communicatifs », « dont le fonctionnement est à bien des égards similaire à celui des énoncés langagiers. » (Kerbrat-Orecchioni, $2004: 31$ ). 